\title{
Classes logarithmiques des corps totalement réels
}

\author{
par \\ Jean-François Jaulent (Talence)
}

1. Position du problème. Dans sa formulation primitive (cf. [3]), la première conjecture de Gross postule, à l'image de celle de Leopoldt, la nonnullité d'un certain régulateur construit sur la partie imaginaire du groupe des $\ell$-unités d'un corps de nombres. Sous sa forme généralisée (cf. [5]), elle n'est rien d'autre en fait que la transposition naturelle en termes $\ell$-adiques du théorème de Dirichlet sur le groupe des unités attaché à un corps de nombres algébriques $K$.

De façon classique, en effet, si $K$ possède $r$ plongements réels $\sigma_{v}$ et $2 c$ plongements complexes $\sigma_{v}$ et $\bar{\sigma}_{v}$ (i.e. $r+c$ places à l'infini), le plongement logarithmique à l'infini

$$
\mathcal{L}_{\infty} \mid K^{\times} \rightarrow \bigoplus_{v \mid \infty} \mathbb{R}=\mathbb{R}^{r+c}
$$

s'obtient en associant à chaque $x$ de $K^{\times}$la famille indexée par les places à l'infini

$$
\mathcal{L}_{\infty}(x)=\left(\log _{\infty}|x|_{v}\right)_{v \mid \infty}=\left(\log _{\infty} N_{K_{v} / \mathbb{R}}(x)\right)_{v \mid \infty},
$$

où l'on a posé $|x|_{v}=\left|\sigma_{v}(x)\right|$ si $v$ est réelle, $|x|_{v}=\left|\sigma_{v}(x)\right|^{2}=N_{K_{v} / \mathbb{R}}\left(\sigma_{v}(x)\right)$ si $v$ est complexe, et noté $\log _{\infty}$ l'unique prolongement à $\mathbb{R}^{\times}$du logarithme népérien sur $\mathbb{R}_{+}^{\times}$qui vérifie l'équation fonctionnelle $\log _{\infty}(x y)=\log _{\infty} x+$ $\log _{\infty} y$.

De façon semblable, si le corps $K$ possède $l$ plongements $\ell$-adiques $\sigma_{\mathfrak{l}}$ pour un premier $\ell$ (i.e. $l$ places $\ell$-adiques), le plongement logarithmique $\ell$-adique

$$
\mathcal{L}_{\ell} \mid K^{\times} \rightarrow \bigoplus_{\mathfrak{\imath} \mid \ell} \mathbb{Q}_{\ell}=\mathbb{Q}_{\ell}^{l}
$$

s'obtient en associant à un $x$ de $K^{\times}$la famille indexée par les places $\ell$-adiques

$$
\mathcal{L}_{\ell}(x)=\left(\log _{\ell}|x|_{\mathfrak{r}}\right)_{\mathfrak{r} \mid \ell}=\left(\log _{\ell} N_{K_{\mathfrak{r}} / \mathbb{Q}_{\ell}}(x)\right)_{\mathfrak{r} \mid \ell}
$$

2000 Mathematics Subject Classification: 11R37, 11R23, 11J86. 
où l'on a noté $|x|_{\mathfrak{r}}=\left|\sigma_{\mathfrak{l}}(x)\right|$ la projection de $N_{K_{\mathfrak{l}} / \mathbb{Q}_{\ell}}(x)=N_{K_{\mathfrak{l}} / \mathbb{Q}_{\ell}}\left(\sigma_{\mathfrak{l}}(x)\right)$ sur le sous-groupe principal $U_{\ell}^{1}$ de $\mathbb{Q}_{\ell}^{\times}=\mu_{\ell}^{\circ} U_{\ell}^{1} \ell^{\mathbb{Z}}$, et $\log _{\ell}$ l'unique prolongement à $\mathbb{Q}_{\ell}^{\times}$du logarithme $\ell$-adique qui vérifie la convention d'Iwasawa $\log _{\ell} \ell=1$ et l'équation fonctionnelle $\log _{\ell}(x y)=\log _{\ell} x+\log _{\ell} y$.

Si donc l'on remarque que le groupe des $\ell$-unités totalement positives

$$
E_{K}^{\prime}=\left\{x \in K^{\times} \mid v_{\mathfrak{p}}(x)=0 \forall \mathfrak{p} \nmid \ell\right\}
$$

est le pendant $\ell$-adique de celui des unités $E_{K}=\left\{x \in K^{\times} \mid v_{\mathfrak{p}}(x)=0\right.$ $\forall \mathfrak{p} \nmid \infty\}$, on voit que le classique théorème de Dirichlet, qui affirme que le $\mathbb{R}$-espace engendré par l'image $\mathcal{L}_{\infty}\left(E_{K}\right)$ de $E_{K}$ dans $\mathbb{R}^{r+c}$ est l'hyperplan $H_{\infty}=\left\{\left(x_{1}, \ldots, x_{r+c}\right) \in \mathbb{R}^{r+c} \mid \sum x_{i}=0\right\}$, se traduit dans le contexte $\ell$-adique par l'énoncé suivant, qui constitue précisément la conjecture de Gross généralisée :

Conjecture. Avec les notations ci-dessus, le $\mathbb{Q}_{\ell}$-espace engendré par l'image $\mathcal{L}_{\ell}\left(E_{K}^{\prime}\right)$ dans $\mathbb{Q}_{\ell}^{l}$ du groupe des $\ell$-unités (positives) $E_{K}^{\prime}$ d'un corps de nombres $K$ ayant $l$ places $\ell$-adiques est l'hyperplan $H_{\ell}=\left\{\left(x_{1}, \ldots, x_{l}\right) \in\right.$ $\left.\mathbb{Q}_{\ell}^{l} \mid \sum x_{i}=0\right\}$.

Et tout le problème est d'établir ce résultat en l'absence du secours de la géométrie des nombres. Avant d'en proposer une preuve pour certains corps par des méthodes de transcendance, nous en donnons d'abord la traduction en termes de classes logarithmiques, qui justifie le titre de l'article.

2. Classes logarithmiques. Le $\ell$-groupe des classes logarithmiques d'un corps de nombres $K$ peut se définir comme le quotient du $\mathbb{Z}_{\ell \text {-module }}$ $\widetilde{\mathcal{D} \ell_{K}}$ des diviseurs logarithmiques de degré nul (i.e. du sous-module du $\mathbb{Z}_{\ell^{-}}$ module libre $\mathcal{D} \ell_{K}=\bigoplus_{\mathfrak{p}} \mathbb{Z}_{\ell} \mathfrak{p}$ construit sur les places finies de $K$ formé des diviseurs $\delta=\sum_{\mathfrak{p}} n_{\mathfrak{p}} \mathfrak{p}$ de degré total $\operatorname{deg} \delta=\sum_{\mathfrak{p}} n_{\mathfrak{p}} \operatorname{deg} \mathfrak{p}=0$ ) par le sous-module principal $\widetilde{\mathcal{P}}_{K}$ obtenu en envoyant dans $\widetilde{\mathcal{D} \ell_{K}}$ le tensorisé $\mathcal{R}_{K}=\mathbb{Z}_{\ell} \otimes_{\mathbb{Z}} K^{\times}$du groupe multiplicatif de $K^{\times}$au moyen des logarithmes d'Iwasawa des valeurs absolues $\ell$-adiques. Avec les notations de la théorie $\ell$-adique du corps de classes, le $\ell$-groupe des diviseurs logarithmiques est le quotient $\mathcal{D} \ell_{K}=\mathcal{J}_{K} / \prod_{\mathfrak{p}} \widetilde{\mathcal{U}}_{\mathfrak{p}}$ du $\ell$-adifié $\mathcal{J}_{K}=\prod_{\mathfrak{p}}^{\text {res }} \mathcal{R}_{\mathfrak{p}}$ du groupe des idèles de $K$ par le sous-groupe $\prod_{\mathfrak{p}} \widetilde{\mathcal{U}}_{\mathfrak{p}}$ des normes cyclotomiques locales; le sous-module des diviseurs logarithmiques de degré nul est le quotient $\widetilde{\mathcal{J}}_{K} / \prod_{\mathfrak{p}} \widetilde{\mathcal{U}}_{\mathfrak{p}}$, où $\widetilde{\mathcal{J}}_{K}$ est le sous-groupe de $\mathcal{J}_{K}$ associé par la théorie à la $\mathbb{Z}_{\ell}$-extension cyclotomique $K^{\mathrm{c}}$ de $K$; et $\widetilde{\mathcal{P} \ell_{K}}$ n'est autre que l'image dans $\mathcal{D} \ell_{K}$ du sous-module $\mathcal{R}_{K}$ des idèles principaux (cf. [6]). Le quotient

$$
\widetilde{\mathcal{C} \ell_{K}}=\widetilde{\mathcal{D} \ell_{K}} / \widetilde{\mathcal{P}} \ell_{K} \simeq \widetilde{\mathcal{J}}_{K} / \prod_{\mathfrak{p}} \widetilde{\mathcal{U}}_{\mathfrak{p}} \mathcal{R}_{K}
$$


s'identifie donc au groupe de Galois relatif $\operatorname{Gal}\left(K^{\text {lc }} / K^{\mathrm{c}}\right)$ où $K^{\mathrm{c}}$ est le $\mathbb{Z}_{\ell^{-}}$ extension cyclotomique de $K$ et $K^{\text {lc }}$ la pro- $\ell$-extension abélienne maximale de $K$ qui est localement cyclotomique partout (i.e. complètement décomposée sur $K^{\mathrm{c}}$ en chacune de ses places).

Du point de vue de la théorie d'Iwasawa, le groupe $\widetilde{\mathcal{C} \ell_{K}}$ n'est rien d'autre que le quotient des $\ell$-genres attaché au groupe de Galois $\mathcal{C}_{\infty}^{\prime}=\operatorname{Gal}\left(C_{\infty}^{\prime} / K^{\mathrm{c}}\right)$ de la pro- $\ell$-extension abélienne complétement décomposée maximale $C_{\infty}^{\prime}$ de $K^{\mathrm{c}}$

$$
\widetilde{\mathcal{C}}_{K} \simeq{ }_{I} \mathcal{C}_{\infty}^{\prime}=\mathcal{C}_{\infty}^{\prime} / \mathcal{C}_{\infty}^{\prime \gamma-1}
$$

relativement au groupe procyclique $\Gamma=\operatorname{Gal}\left(K^{\mathrm{c}} / K\right)=\gamma^{\mathbb{Z}_{\ell}}:$ la $\mathbb{Z}_{\ell}$-extension cyclotomique $K^{\mathrm{c}}$ est, en effet, la réunion $K^{\mathrm{c}}=K_{\infty}=\bigcup_{n \in \mathbb{N}} K_{n}$ de ses étages finis et sa pro- $\ell$-extension abélienne maximale qui est complètement décomposée en chacune de ses places est la réunion $C_{\infty}^{\prime}=\bigcup_{n \in \mathbb{N}} C_{n}^{\prime}$ des $\ell$-extensions abéliennes respectives $C_{n}^{\prime}$ des $K_{n}$ qui sont non ramifiées $\ell$ décomposées (i.e. non ramifiées partout et complètement décomposées aux places au-dessus de $\ell$ ) et maximales sous ces conditions ; dans l'isomorphisme du corps de classes les groupes de Galois $\operatorname{Gal}\left(C_{n}^{\prime} / K_{n}\right)$ s'identifient aux $\ell$ groupes de $\ell$-classes d'idéaux respectifs $\mathcal{C} \ell_{n}^{\prime}$ des corps $K_{n}$ (i.e. aux quotients des $\ell$-groupes de classes au sens ordinaire par les sous-groupes engendrés par les classes des places au-dessus de $\ell)$ et le groupe $\operatorname{Gal}\left(C_{\infty}^{\prime} / K_{\infty}\right)$ à la limite projective considérée par Greenberg (cf. [4]) :

$$
\mathcal{C}_{\infty}^{\prime}=\varliminf_{\longleftarrow} \mathcal{C} \ell_{n}^{\prime} .
$$

Enfin, comme $K^{\text {lc }}$ est la plus grande sous-extension de $C_{n}^{\prime}$ qui est abélienne sur $K$, le groupe des classes logarithmiques $\widetilde{\mathcal{C} \ell_{K}}=\operatorname{Gal}\left(K^{\text {lc }} / K^{\mathrm{c}}\right)$ est aussi le plus grand quotient ${ }_{\Gamma} \mathcal{C}_{\infty}^{\prime}$ de $\mathcal{C}_{\infty}^{\prime}$ sur lequel $\Gamma$ agit trivialement, ce qui explique, en particulier, les liens entre $\ell$-groupes de classes logarithmiques et $\ell$-parties des noyaux sauvages de la $K$-théorie.

Comme expliqué dans [6], la conjecture de Gross généralisée postule précisément la finitude du groupe $\widetilde{\mathcal{C} \ell_{K}}$. Pour chaque place $\mathfrak{l}$ de $K$ au-dessus de $\ell$, notons en effet $\widetilde{v}_{\mathfrak{l}}=\log ||_{\mathfrak{r}} / \operatorname{deg} \mathfrak{l}$ la valuation logarithmique attachée à $\mathfrak{l}$; il vient :

Proposition. Les trois assertions suivantes sont équivalentes:

(i) le l-groupe $\widetilde{\mathcal{C} \ell_{K}}$ des classes logarithmiques de $K$ est fini;

(ii) l'application $\left(\widetilde{v}_{\mathfrak{l}}\right)_{\mathfrak{l} \mid \ell}$ envoie le tensorisé $\mathcal{E}_{K}^{\prime}=\mathbb{Z}_{\ell} \otimes_{\mathbb{Z}} E_{K}^{\prime}$ du groupe des $\ell$-unités de $K$ sur un sous-groupe d'indice fini du $\mathbb{Z}_{\ell}$-module $\widetilde{\bigoplus}_{\mathfrak{l} \mid \ell} \mathbb{Z}_{\ell} \mathfrak{l}$ formé des diviseurs de degré nul construits sur les places au-dessus de $\ell$;

(iii) le corps $K$ satisfait la conjecture de Gross généralisée pour le premier $\ell$. 
Preuve. L'équivalence des deux premières assertions résulte de l'exactitude de la suite canonique (cf. $[6, \S 3]$ )

$$
\mathcal{E}_{K}^{\prime} \rightarrow \widetilde{\bigoplus_{\mathfrak{l} \ell}} \mathbb{Z}_{\ell} \mathfrak{l} \rightarrow \widetilde{\mathcal{C} \ell_{K}} \rightarrow \mathcal{C} \ell_{K}^{\prime}
$$

et du fait que le $\ell$-groupe des $\ell$-classes d'idéaux $\mathcal{C} \ell_{K}^{\prime}$ qui apparaît à droite est fini. L'équivalence des deux dernières est immédiate.

3. Énoncé du Théorème principal. Le résultat principal de cette note peut s'énoncer comme suit :

THÉORÈME. Soient $K$ un corps de nombres absolument galoisien et $\ell$ un nombre premier dont le sous-groupe de décomposition $D_{\ell}$ est distingué dans $G=\operatorname{Gal}(K / \mathbb{Q})$. Supposons réunies les deux conditions suivantes:

(i) le sous-corps de décomposition $H=K^{G}$ est totalement réel et

(ii) l'algèbre de groupe $\mathbb{Q}_{\ell}\left[G / D_{\ell}\right]$ n'a pas de facteur $M_{n}(D)$ avec $n>2$.

Alors le corps $K$ vérifie la conjecture de Gross généralisée pour le nombre premier $\ell$; autrement dit, le $\ell$-groupe $\widetilde{\mathcal{C}}_{K}$ des classes logarithmiques de $K$ est fini.

REMARQue. Il est facile de voir que la conjecture de Gross est héréditaire en ce sens qu'elle est vérifiée dans un corps de nombres $H$ dès qu'elle l'est dans l'un de ses sur-corps de degré fini $K$ : en effet, si elle était en défaut dans $H$, la pro- $\ell$-extension abélienne localement cyclotomique maximale $H^{\text {lc }}$ de $H$ serait de degré infini sur la $\mathbb{Z}_{\ell^{-}}$extension cyclotomique $H^{\mathrm{c}}$ et le compositum $K H^{\text {lc }}$ serait alors une pro- $\ell$-extension abélienne localement cyclotomique de $K$ de degré infini sur $K^{\mathrm{c}}=K H^{\mathrm{c}}$; en particulier $\widetilde{\mathcal{C} \ell_{K}}=\operatorname{Gal}\left(K^{\mathrm{lc}} / K^{\mathrm{c}}\right)$ serait également infini. Sous les hypothèses du théorème, la conjecture de Gross généralisée se teste donc en fait dans le sous-corps de décomposition $H$ de la place $\ell$. Autrement dit :

Corollaire. La conjecture de Gross généralisée pour un premier $\ell$ vaut dans tout corps de nombres absolument galoisien $K$ qui est totalement réel et totalement $\ell$-adique (i.e. dont le complété en chaque place $\ell$-adique est égal $\left.\grave{a} \mathbb{Q}_{\ell}\right)$ dès que l'algèbre de groupe $\mathbb{Q}_{\ell}[\operatorname{Gal}(K / \mathbb{Q})]$ ne contient pas d'algèbre de matrices sur un corps gauche $M_{n}(D)$ avec $n>2$ et, plus généralement, dans toute extension finie $L$ d'un tel corps où les places $\ell$-adiques de $K$ ne se décomposent pas.

Preuve du Corollaire. Le premier cas du corollaire correspond au cas particulier $K=H$. Pour voir que ce résultat vaut encore dans toute extension finie $L$ de $K$ dans laquelle les places $\ell$-adiques de $K$ ne se décomposent pas, il suffit d'observer que l'hyperplan $H_{\ell}(L)=H_{\ell}(K)$ est alors $\mathbb{Q}_{\ell}$-engendré par l'image logarithmique de $E_{K}^{\prime}$ donc a fortiori par celle de $E_{L}^{\prime}$. 
Remarque. De même qu'il est assez naturel d'étudier la conjecture de Leopoldt pour les corps totalement réels, de même la question de la conjecture de Gross se pose naturellement d'abord pour les corps totalement $\ell$-adiques (au sens du Corollaire). Si l'on met alors de côté les approches algébriques, qui ne permettent de conclure que dans des cas particuliers, force est de constater que les méthodes transcendantes, en dépit de leurs récents progrès, ne réussissent à établir la conjecture de Leopoldt pour les corps galoisiens réels que dans le cas où l'algèbre de Galois $\mathbb{Q}_{\ell}[G]$ est un produit direct de corps (i.e. lorsque les caractères $\ell$-adiques irréductibles de $G$ sont de multiplicité au plus 1 dans le caractère régulier). La condition de réalité (i) du Théorème est donc le prix à payer pour pouvoir appliquer avec succès les résultats de transcendance sous l'hypothèse plus faible (ii) où sont acceptés des facteurs irréductibles de multiplicité 2 .

4. Preuve du Théorème principal. Soient donc $K$ une extension galoisienne réelle du corps des rationnels, $G$ son groupe de Galois et $\ell$ un nombre premier vérifiant les hypothèses du Théorème, que nous pouvons supposer, sans perte de généralité, complètement décomposé.

Puisque le corps $K$ est réputé (totalement) réel, nous savons, par le théorème de représentation de Herbrand, que le groupe $E_{K}^{+}$des unités totalement positives est un $\mathbb{Z}[G]$-module de caractère $\chi_{\text {aug }}=\chi_{\text {reg }}-1$ et, puisque $K$ est réputé aussi totalement $\ell$-adique, il suit que le groupe $E_{K}^{\prime}$ des $\ell$-unités totalement positives a lui pour caractère $\chi_{\text {reg }}+\chi_{\text {aug }}=2 \chi_{\text {reg }}-1$. Cela étant :

Lemme D'indépendance. Sous les hypothèses du Théorème, le $\mathbb{Z}_{\ell}[G]$ module $\mathcal{L}\left(\mathcal{E}_{K}^{\prime}\right)$ engendré dans $K_{\ell}=\bigoplus_{\mathfrak{l} \mid \ell} K_{\mathfrak{l}}=\bigoplus_{\mathfrak{l} \mid \ell} \mathbb{Q}_{\ell}$ par les logarithmes d'Iwasawa des éléments de $E_{K}^{\prime}$ a pour caractère $\chi_{\mathcal{L}\left(\mathcal{E}^{\prime}\right)}=\chi_{\mathrm{reg}}-1$.

Preuve du Lemme. Évidemment, $\chi_{\mathcal{L}\left(\mathcal{E}^{\prime}\right)} \leq \chi_{\text {aug }}$ puisque l'image $\mathcal{L}_{\ell}\left(\mathcal{E}^{\prime}\right)$ du $\ell$-adifié $\mathcal{E}_{K}^{\prime}=\mathbb{Z}_{\ell} \otimes_{\mathbb{Z}} E_{K}^{\prime}$ du groupe des $\ell$-unités totalement positives $E_{K}^{\prime}$ est contenue dans l'hyperplan $H_{\ell}$ qui est bien un $\mathbb{Q}_{\ell}[G]$-module de caractère $\chi_{\text {aug }}$; et tout le problème est d'établir l'inégalité opposée $: \chi_{\mathcal{L}\left(\mathcal{E}^{\prime}\right)} \geq \chi_{\text {reg }}-1$, i.e. de vérifier que les caractères $\mathbb{Q}_{\ell}$-irréductibles $\varphi$ représentés dans $\chi_{\text {aug }}$ le sont encore avec la même multiplicité dans $\chi_{\mathcal{L}\left(\mathcal{E}^{\prime}\right)}$.

Distinguons donc deux cas, suivant que cette multiplicité vaut 1 ou 2 :

(i) Si $\varphi$ est représenté une seule fois dans $\chi_{\text {aug }}$, le théorème de BakerBrumer (cf. [1]) nous suffit pour conclure : prenons, en effet, $\varepsilon \in E_{K}^{+}$engendrant un sous- $\mathbb{Z}[G]$-module d'indice fini de $E_{K}^{+} ;$l'élément $\varepsilon_{\varphi}=\varepsilon^{\sum_{\tau \in G} \varphi(\tau) \tau^{-1}}$ de $\mathcal{E}_{K}^{\prime}$ engendre alors un $\mathbb{Z}_{\ell}[G]$-module isotypique de caractère $\varphi$ et il en est de même pour son image $\mathcal{L}_{\ell}\left(\varepsilon_{\varphi}\right)$ dans $K_{\ell}$. Observons au passage que, si $\varphi$ prend ses valeurs dans $\mathbb{Z}$, l'élément $\varepsilon_{\varphi}$ est dans $E_{K}^{\prime}$ et l'inégalité $\mathcal{L}_{\ell}\left(\varepsilon_{\varphi}\right) \neq 0$ est alors évidente, indépendamment de tout argument de transcendance. 
(ii) $\mathrm{Si} \varphi$ est représenté deux fois dans $\chi_{\text {aug }}$, le caractère $\chi_{\mathcal{E}^{\prime}}$ contient $r_{\varphi}=4$ fois le caractère $\varphi$, tandis que le caractère régulier $\chi_{\text {reg }}$ le contient $d_{\varphi}=2$ fois. L'indice $k=\left[r_{\varphi} / d_{\varphi}\right]$ introduit par M. Emsalem (cf. [2]) est ici égal à 2 et les raffinements du théorème de M. Waldschmidt (cf. [9]) donnés par M. Laurent (cf. [7, th. 1]) et D. Roy (cf. [8, th. 7]) nous assurent que $\chi_{\mathcal{L}\left(\mathcal{E}^{\prime}\right)}$ contient $\varrho_{\varphi} \geq r_{\varphi} d_{\varphi} /\left(r_{\varphi}+d_{\varphi}\right)=8 / 6>1$ fois le caractère $\varphi$; d'où le résultat.

REMARQUE. Le lecteur attentif aura peut être remarqué que nous avons raisonné sur la décomposition irréductible du caractère $\chi_{\mathcal{E}^{\prime}}$, alors même que les résultats de transcendance que nous utilisons (notamment [7] et [8]) font intervenir, eux, la décomposition absolument irréductible des caractères considérés.

Expliquons brièvement pourquoi cette distinction est sans conséquence : écrivons, en effet,

$$
\varphi=n_{\varphi} \sum_{\phi \mid \varphi} \phi
$$

la décomposition absolument irréductible du caractère irréductible $\varphi$. Dans les décompositions respectives du caractère d'augmentation $\chi_{\text {aug }}$ et du caractère $\chi_{\mathcal{E}^{\prime}}$, nous sommes alors amenés à remplacer $r_{\varphi}$ par le produit $r_{\phi}=$ $n_{\varphi} r_{\varphi}$ et $d_{\varphi}$ par le produit $d_{\phi}=n_{\varphi} d_{\varphi}$, de sorte que nous avons toujours $\left[r_{\phi} / d_{\phi}\right]=2$, et les résultats de transcendance invoqués nous assurent que $\chi_{\mathcal{L}\left(\mathcal{E}^{\prime}\right)}$ contient $\varrho_{\phi} \geq r_{\phi} d_{\phi} /\left(r_{\phi}+d_{\phi}\right)=8 n_{\varphi} / 6>n_{\varphi}$ fois le caractère $\phi$, donc, comme attendu, $\varrho_{\varphi}=\varrho_{\phi} / n_{\varphi}>1$ fois le caractère $\varphi$.

Il est évidemment possible d'énoncer ce qui précède caractère par caractère :

SCOLIE. Soient $K$ un corps de nombres absolument galoisien et $\chi_{\mathrm{reg}}=$ $\sum n_{\varphi} \varphi$ la décomposition canonique du caractère régulier de $G=\operatorname{Gal}(K / \mathbb{Q})$ comme somme de caractères $\mathbb{Q}_{\ell}$-irréductibles, puis $\chi_{\ell}=\sum n_{\varphi}^{\ell} \varphi\left(\right.$ resp. $\chi_{\infty}=$ $\left.\sum n_{\varphi}^{\infty} \varphi\right)$ celle du caractère de l'induite à $G$ de la représentation unité du sous-groupe de décomposition de l'une quelconque des places $\ell$-adiques (resp. à l'infini) de $K$. Pour chaque caractère $\varphi$ qui vérifie les conditions

$$
n_{\varphi}^{\ell}=n_{\varphi}^{\infty}=n_{\varphi} \leq 2
$$

la conjecture de Gross généralisée est vraie en $\varphi$ pour le nombre premier $\ell$ (en ce sens que la $\varphi$-composante du caractère $\chi_{\mathcal{L}\left(\mathcal{E}^{\prime}\right)}$ coïncide bien avec celle de $\left.\chi_{\ell}-1\right)$.

En exprimant que les hypothèses $(*)$ sont satisfaites pour chaque facteur irréductible $\varphi$ de $\chi_{\ell}-1$, on voit que l'identité $n_{\varphi}^{\ell}=n_{\varphi}$ s'écrit globalement $\chi_{\ell} \wedge\left(\chi_{\text {reg }}-\chi_{\ell}\right)=0$, tandis que l'inégalité $n_{\varphi}^{\ell} \leq n_{\varphi}^{\infty}$ donne $\chi_{\ell} \leq \chi_{\infty}$; la première condition entraîne en fait que les sous-groupes de décomposition 
des places $\ell$-adiques soient distingués dans $G$ et la seconde implique alors que leur sous-corps commun de décomposition soit totalement réel. On retombe donc bien sur le Théorème principal.

Bien entendu, le résultat obtenu est banalement vrai si le corps considéré satisfait la conjecture de Leopoldt.

\section{Références}

[1] A. Brumer, On the units of algebraic number fields, Mathematika 14 (1967), 121-124.

[2] M. Emsalem, Rang p-adique de groupes de $S$-unités d'un corps de nombres, C. R. Acad. Sci. Paris Sér. I 297 (1983), 225-227.

[3] L. J. Federer and B. H. Gross (with an appendix by W. Sinnott), Regulators and Iwasawa modules, Invent. Math. 62 (1981), 443-457.

[4] R. Greenberg, On a certain $\ell$-adic representation, ibid. 21 (1973), 117-124.

[5] J.-F. Jaulent, Sur l'indépendance $\ell$-adique de nombres algébriques, J. Number Theory 20 (1985), 149-158.

[6] -, Classes logarithmiques des corps de nombres, J. Théor. Nombres Bordeaux 6 (1985), 301-325.

[7] M. Laurent, Rang p-adique d'unités et action de groupes, J. Reine Angew. Math. 399 (1989), 81-108.

[8] D. Roy, Matrices whose coefficients are linear forms in logarithms, J. Number Theory 41 (1992), 22-47.

[9] M. Waldschmidt, A lower bound for the p-adic rank of the units of an algebraic number field, dans : Topics in Classical Number Theory, Colloq. Math. Soc. János Bolyai 34, North-Holland, 1981, 1617-1650.

Institut de Mathématiques

Université Bordeaux I

351, cours de la Libération

F-33405 Talence Cedex, France

E-mail: jaulent@math.u-bordeaux.fr

Reçu le 12.5.1998

et révisé le 23.11.2001 